\title{
W. KREISELS SCHWEIZERISCHER RELIEFKATALOG
}

\author{
Von Eduard IMHOF
}

\author{
Mit einer Abbildung
}

Die bedeutendste Reliefsammlung der Schweiz und eine der reichhaltigsten überhaupt befindet sich im Alpinen Museum in Bern. Die Museumsleitung betrachtet es daher als ihre Pflicht und Aufgabe, das Reliefwesen und die Reliefbibliographie zu fördern. Im Jahre 1937 erschien als Nr. 6 der «Wissenschaftlichen Mitteilungen» dieses Museums eine ausgezeichnete Arbeitüber "Das topographische Relief in der Schweiz ", verfaßt von FrITz GyGAX. Sie enthielt erstmals eine Katalogisierung schweizer. Reliefs.

Kürzlich wurde als Heft 7 derselben Bücherreihe eine zweite Arbeit herausgegeben: "Schweizerischer Reliefkatalog, ein Uberblick über das schweizerische Reliefwesen", bearbeitet von WILLY KREISEL, Ingenieur der Eidgenössischen Landestopographie ${ }^{1}$. Kreisel ist nicht nur

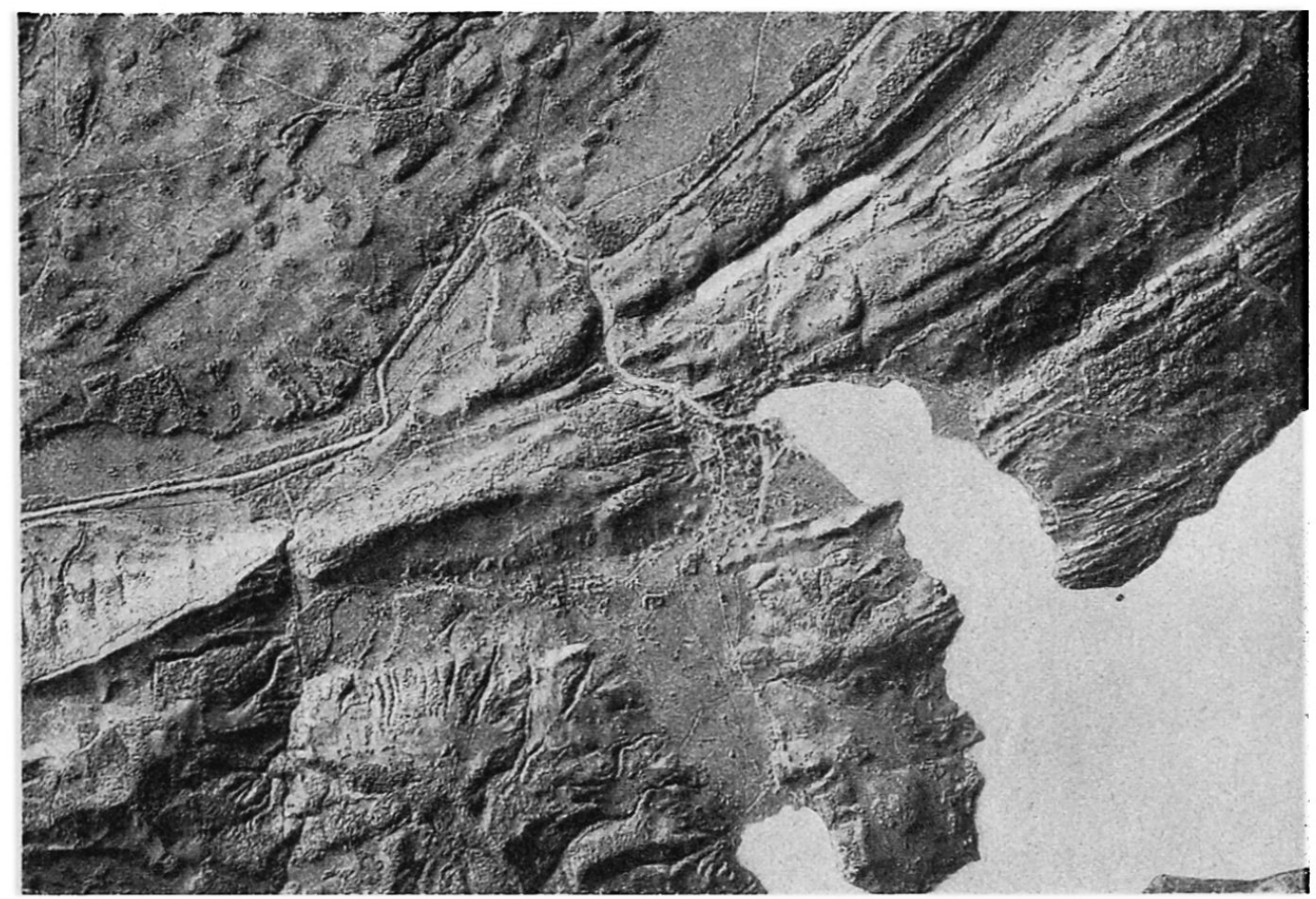

Abbildungsbeispiel: Luzernersee, Relief 1:25000 von Xaver IMFeld, 1889.

Bearbeiter, sondern auch Initiant dieser jüngsten, beachtenswerten und sehr reichhaltigen Inventarisierung. Er wurde unterstützt durch die Eidgenössische Landestopographie. Diese hatte den Bearbeiter mit der Errichtung einer Reliefregistratur beauftragt; sie besorgte überdies den Druck der dem Buche beigegebenen fünf Karten.

Ein erster Teil des Buches gibt Orientierungen und Anregungen über Reliefregistratur und Katalogisierung, ferner über Reliefs und deren Verwendung im allgemeinen. Ein zweiter Teil enthält Verzeichnisse der in der Schweiz vorhandenen Reliefs und ihrer Ersteller. Den Abschluß bildet eine reichhaltige Bibliographie.

Die beigefügten Karten zeigen, getrennt nach Maßstäben, welche Gebiete der Schweiz bisher reliefiert worden sind. Es ist dies, besonders in den Maßstäben 1:25000 und kleiner, erstaunlich viel, wenn man bedenkt, daß fast alle bisherigen Arbeiten privater Initiative zuzusshreiben sind.

Das topographische Relief ist zweifellos das übersichtlichste und vielseitigste Hilfsmittel zur Veranschaulichung von Geländeformen. Nachteilig sind neben zeitraubender Erstellung nur die beschränkten Möglichkeiten der Aufbewahrung oder Aufstellung und die schwerfälligen Transporte. Im Gegensatz zur Karte, die uns begleitet, muß man in der Regel zu ihm hingehen, wie zum Berge selber. Hiezu aber ist die Kenntnis des Vorhandenseins und des Standortes ein erstes Erfordernis. Kreisel gibt durch seinen Katalog diese Orientierung und fördert damit den Reliefgebrauch in hohem Maße.

${ }^{1}$ Barn 1947, 78 Seiten, 14 Abbildungen, 5 Kartenbeilagen. 
Der Verfasser war bestrebt, nicht nur die Reliefs öffentlicher Sammlungen, sondern auch diejenigen aus Privatbesitz in seinem Katalog aufzuführen. Hiezu waren alle Abgüsse ein- und desselben Modelles aufzuspüren. Dies erschwerte die Arbeit ungemein, erhöhte jedoch deren praktischen Nutzen. $W_{\text {ir }}$ erhalten ein eindrückliches Bild von der Unsumme bekannter und verborgener Leistungen, vom Ameisenfleiß zahlreicher Topographen, Geologen und Topoplastiker jeder Art, vor allem auch der vielen Lehrer im Lande herum, die sich aus Idealismus an plastische Geländenachbildungen herangewagt haben.

Dieses Streben nach Vollständigkeit birgt aber auch Nachteile in sich. Nicht jedes topographische Modell ist die Druckerschwärze des Katalogisierens wert. In dieser Hinsicht teilt freilich ein Relief katalog das Schicksal mit manchem Bücherverzeichnis. Je weiter aber eine Reliefkatalogisierung getrieben wird, um so rascher veraltet sie. Ist es nicht eine Tücke des Objekts, da $B$ das sichtbarste und daher bekannteste aller Reliefs in der Schweiz, Simons imposante Plastik der Berner Alpen, unmittelbar nach dem Druck des Kataloges vom Zürcher Hauptbahnhof in die Eidgenössische Technische Hochschule umgezogen ist? Kleinere Modelle in privatem Besitz können entstehen, den Standort wechseln oder verschwinden, ohne die eidgenössische Reliefregistratur im geringsten in Schwingung zu versetzen. Zur Erfassung aller Mutationen müßte ein Kontrollapparat aufgezogen werden, der die ganze Sache nicht wert wärc. Wohl das einzige, jedoch reichlich problematische Mittel zur dauernden Nachführung der Reliefregistratur ist der periodisch wiederkehrende Aufruf in Lehrerzeitungen und Fachzeitschriften. In diesem Sinne möchten wir schon heute alle Reliefersteller und -besitzer nachdrücklich um eine Durchsicht des Kataloges und um Meldung von Unstimmigkeiten an Ingenieur KrEISEL, Eidgenössische Landestopographie, Wabern-Bern, bitten. Es werden hiefür besondere Meldezettel zur Verfügung gestellt.

Die Schweiz stand in der Erstellung topographischer Reliefs bisher an der Spitze. Heute macht das Ausland große Anstrengungen, auf mechanisiertem Wege die Relieferstellung zu fördern und die plastische Geländewiedergabe in den Dienst der Kartographie zu stellen. Wir werden quantitativ solchen Entwicklungen nicht folgen können. Hingegen soll es unser Bestreben sein, qualitativ wie bisher die Führung zu behalten. KreISFLs Katalog lenkt den Blick auf bisher Erreichtes und wird damit zu neuen Anstrengungen anspornen.

\section{NEKROLOGIE}

\section{Hans Schärer, '1904-1947}

Am 10. Dazember 1947 verschied ganz unerwartet in Bandjermasin (Borneo) der schweizerische Missionar und Ethnologe Dr. phil. HANS SCHÄRER in seinem 43. Altersjahre. Geboren am 27. Juni 1904 in Wädenswil, wo er die Primar- und Sekundarschulen besuchte, trat SCHÄRER nach Absolvierung einer dreijährigen Dienstzeit als Stationsgehilfe bei der Schweizerischen Südostbahn im Jahre 1925 ins Seminar der Basler Mission ein, wo er 1932 seine Ausbildung abschloß. Nach vorbereitenden Sprachstudien in Holland und der Teilnahme an einem Tropenkurs in Tübingen trat er noch im gleichen Jahre sein Amt als Missionar in Borneo an, wo er, von seiner Gattin unterstützt, in einsamer Gegend bis 1939 Pionierarbeit geleistet und auf seinen Reisen wertvolles ethnographisches Material gesammeit hat. Dieses bezog sich vor allem auf die Erforschung der uralten religiösen Anschauungen der Eingeborenen von Borneo als seinem eigentlichen Spezialgebiet. Die Verarbeitung dieses Materials erfolgte in Holland, wo SCHÄrER die Kriegszeit an der Universität Leiden verbrachte und im Jahre 1946 bei Prof. Dr. J. P. B. De Josselin DE Jong mit einer ethnologischen Dissertation über "Die Gottesidee bei den Ngadju-Dajak" den Doktorgrad erwarb. Aus jener vom Tode seiner Gattin überschattetcn Zeit stammen mehrere wissenschaftliche Arbeiten, von denen diejenigen über Gottesurteil und Verfluchung bei den Katinganern, die Bedeutung des Menschopfers im dajakischen Totenkult und die Stellung der Frau bei den Ngadju-Dajak hervorgehoben seien. Vor der Geographisch-Ethnographischen Gesellschaft Zürich hielt SCHÄRER im Januar 1946 einen fesselnden Vortrag über die Religion der Dajak, der den Hörern noch in guter Erinnerung ist. Im Herbst desselben Jahres kehrte er in Begleitung seiner zweiten Gattin (einer für Borneo bestimmten Krankenschwester) nach seinem ursprünglichen Tätigkeitsfeld in Borneo zurück, diesmal als Vorsteher des dortigen Missionsfeldes, wo er, sich alte Beziehungen wieder anknüpfend, mit aller Energie für seine Aufgabe einsetzte. Ein gutes Jahr später hat nun ein heimtückisches Fieber den jungen Forscher, der die Ausarbeitung eines umfangreichen und noch nicht ausgewerteten ethnologischen Materials plante, der Arbeit und den Seinen jäh entrissen. Diejenigen, die ihn näher kannten, haben in ihm einen Menschen schätzen gelernt, der dank seiner völkerkundlichen Schulung, frei von Dogmatik, mit offenem Sinn und großem Verständnis an die Bewältigung der ihm gestellten Aufgaben herantrat. Mit ihm ist nicht nur ein ausgezeichneter Missionar, sondern zugleich ein begabter Ethnologe hingegangen, dessen wissenschaftliche Laufbahn zu den schönsten Hoffnungen berechtigte.

A. STEINMANN

\section{NEUIGKEITEN - NOVA}

Zur Methodik der Gebirgssiedlungsforschung. Nachdem 1945 durch die bauliche Sanierung von Hotels und Kurorten eine ausgezeichnete Grundlage der Untersuchung städtischer Alpensiedlungen geschaffen wurde, hat nun in der instruktiv illustrierten Schrift von $\mathrm{H}$. SснӥрвACH "Die technische Ausrüstung im Bergdorf" (Heft 13 dar Bautechnischen Reihe der Schriften zur Frage der Arbeits- 